\title{
As unhas de gel potenciam o número e/ou gravidade dos acidentes de trabalho?- De Scoping Review a Desafio lançado aos Leitores...
}

rpso.pt/as-unhas-de-gel-potenciam-o-numero-e-ou-gravidade-dos-acidentes-de-trabalho-de-scoping10.31252/RPSO.21.03.2019

GEL/ EXTENSION NAILS POTENTIATE THE NUMBER AND/ OR GRAVITY OF WORK ACCIDENTS? - FROM SCOPING REVIEW TO CHALLENGING THE READERS...

Autores: Santos, M(1); Almeida, A(2); Lopes C(3), Oliveira T(4).

\section{RESUMO}

\section{Introdução e objetivo}

Ao longo do desenrolar da prática profissional em contexto da Saúde Ocupacional, por várias vezes os autores se questionaram se existiria alguma relação entre o uso de unhas de gel/ extensões de unhas e Acidentes de Trabalho, sobretudo quando presentes algumas tarefas laborais manuais, que exijam destreza e coordenação e/ ou com risco de atracamento, por vezes na interação com algumas máquinas.

\section{Metodologia}

Com o objetivo de realizar uma Scopping Review, foram consideradas os seguintes motores de busca/ bases de dados: Scopus; PubMed/ Medline; Web of Science; Science Direct; Academic Search Ultimate; CINALH; Database of Abstracts and Reviews; Central Register of Controlled Trials; Cochrane Database of Systematic Reviews; Nursing and Allied Health Collection; MedicLatina e RCAAP.

\section{Resultados}

Nenhum dos artigos selecionados nas pesquisas descritas foi capaz de responder à questão de investigação.

\section{Discussão e Conclusão}

Uma vez que não se encontraram quaisquer dados sobre este tema, a Direção da Revista Portuguesa de Saúde Ocupacional lança mais um desafio aos seus leitores, no sentido de tentar aliciar uma Equipa de Saúde Ocupacional, que tenha acesso a uma 
amostra de trabalhadoras significativa, em postos de trabalho adequados a esta investigação. Pretender-se-ia tentar correlacionar o número e gravidade dos Acidentes de Trabalho ao longo dos últimos anos, nas trabalhadoras do sexo feminino, que usem unhas de gel, versus trabalhadoras com tarefas equivalentes, mas sem esses adereços; podendo fazê-lo com total autonomia (desde a recolha das autorizações, elaboração do instrumento para recolha de dados, inserção dos mesmos em SPSS, tratamento estatístico, redação de artigo e submissão para publicação) ou apenas recolhendo as autorizações/ dados e inserindo os mesmos em programa estatístico, assumindo alguns elementos da Direção da Revista as restantes etapas, em parceria.

PALAVRAS-CHAVE: unhas de gel, extensões de unhas, acidentes de trabalho, acidentes laborais.

\section{ABSTRACT}

\section{Introduction and objective}

In the context of Occupational Health practice, the authors have questioned whether there is any relationship between the use of gel nails/ nail extensions and Work Accidents, especially when there are some manual labor tasks that require coordination and/ or interaction with some dangerous machines.

\section{Methodology}

In order to carry out a Scopping Review, the following databases were considered: Scopus; PubMed/ Medline; Web of Science; Science Direct; Academic Search Ultimate; CINALH; Database of Abstracts and Reviews; Central Register of Controlled Trials; Cochrane Database of Systematic Reviews; Nursing and Allied Health Collection; MedicLatina and RCAAP.

\section{Results}

None of the articles selected in the surveys described was able to answer the research question.

\section{Discussion and Conclusion}

Since no data on this topic were found, the RPSO on line launches another challenge to its readers, in order to try to motivate an Occupational Health Team, with access to a significant sample of female workers, in jobs appropriate to this research. It would be pretended to try to correlate the number and severity of Work Accidents during the last years, with workers that use nail extensions, versus workers with equivalent tasks, but without these artificial nails; it could be everything with autonomy (from the collection of authorizations, preparation of the instrument for data collection, insertion of the data into SPSS, statistical treatment, article writing and submission for publication) or with our help, just collecting the authorizations and the data, assuming some elements of the Direction of the Magazine the remaining stages, in partnership. 
KEYWORDS: gel nails, extensions of nails, work accidents, occupational accidents.

\section{INTRODUÇÃO E OBJETIVO}

Ao longo do desenrolar da prática profissional em contexto da Saúde Ocupacional, por várias vezes os autores se questionaram se existiria alguma relação entre o uso de unhas de gel/ extensões de unhas e Acidentes de Trabalho, sobretudo quando presentes algumas tarefas laborais manuais, que exijam destreza e coordenação e/ ou com risco de atracamento, por vezes na interação com algumas máquinas.

A pergunta de investigação considerada foi: "As unhas de gel/ extensões de unhas potenciam o número ou gravidade dos Acidentes Laborais?"

\section{METODOLOGIA}

Realizaram-se pesquisas informais prévias sobre o tema e percebeu-se que a literatura é muito, muito escassa, por isso, os autores optaram por não fazer restrições significativas associadas a ano de publicação, tipo de estudo, robustez metodológica, língua ou acesso imediato a texto completo.

Como critérios de inclusão consideraram-se:

- publicação até fevereiro de 2019

- idade igual ou superior a 18 anos

- sexo feminino

Como critérios de exclusão foram assumidos:

estudos não pertinentes para o objetivo da revisão, ou seja, que não respondam à questão de investigação.

Foram consideradas os seguintes motores de busca/ bases de dados: Scopus; PubMed/ Medline; Web of Science; Science Direct; Academic Search Ultimate; CINALH; Database of Abstracts and Reviews; Central Register of Controlled Trials; Cochrane Database of Systematic Reviews; Nursing and Allied Health Collection; MedicLatina e RCAAP.

\section{RESULTADOS}

Nos seis quadros seguintes estão resumidos os resultados obtidos, na interação das palavras- chave escolhidas e com as bases de dados/ motores de busca considerados.

Quadro 1: Resumo dos dados mais relevantes associados à pesquisa no motor de busca Academic Search Ultimate 
Data

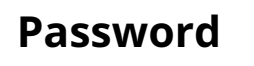

Password $2 \quad N^{\circ}$ de

$N^{\circ} d a$

Pesquisa

1

e seguintes,

caso

documentos

obtidos

pesquisa efetuada

existam

2019/02/25 Nails

\begin{tabular}{llll} 
& 11.020 & 1 & não \\
\hline Gel & 142 & 2 & $\begin{array}{l}\text { Inicialmente } \\
\text { não; depois } \\
\text { sim }\end{array}$ \\
\hline and Accidents & 1 & 3 & sim \\
\hline Extensions & 112 & 4 & $\begin{array}{l}\text { Inicialmente } \\
\text { não; depois } \\
\text { sim }\end{array}$ \\
\hline and Accidents & 4 & 5 & sim
\end{tabular}

Quadro 2: Resumo dos dados mais relevantes associados à pesquisa no motor de busca Science Direct

\begin{tabular}{llllll} 
Data & $\begin{array}{l}\text { Password } \\
\mathbf{1}\end{array}$ & $\begin{array}{l}\text { Password 2 e } \\
\text { seguintes, caso } \\
\text { existam }\end{array}$ & $\begin{array}{l}\mathbf{N}^{\circ} \text { de } \\
\text { documentos } \\
\text { obtidos }\end{array}$ & $\begin{array}{l}\mathbf{N}^{\circ} \text { da } \\
\text { pesquisa }\end{array}$ & $\begin{array}{l}\text { Pesquisa } \\
\text { efetuada } \\
\text { ou não }\end{array}$ \\
\hline 2019/02/25 Nails & 40.271 & 6 & não \\
\cline { 2 - 5 } & & 5.209 & 7 & não \\
\hline Gel & 1.189 & 8 & não \\
\hline and Extensions & and Accidents & 160 & 9 & não \\
\hline and Work & 124 & 10 & sim
\end{tabular}

Quadro 3: Resumo dos dados mais relevantes associados à pesquisa no motor de busca SCOPUS

\begin{tabular}{llllll} 
Data & $\begin{array}{l}\text { Password } \\
\mathbf{1}\end{array}$ & $\begin{array}{l}\text { Password 2 e } \\
\text { seguintes, caso } \\
\text { existam }\end{array}$ & $\begin{array}{l}\mathbf{N}^{\circ} \text { de } \\
\text { documentos } \\
\text { obtidos }\end{array}$ & $\begin{array}{l}\mathbf{N}^{\circ} \text { da } \\
\text { pesquisa }\end{array}$ & $\begin{array}{l}\text { Pesquisa } \\
\text { efetuada } \\
\text { ou não }\end{array}$ \\
\hline 2019/02/25 Nails & & 23.603 & 11 & não \\
\cline { 3 - 6 } & & Gel & 651 & 12 & não
\end{tabular}




\begin{tabular}{lccc}
\hline and Accidents & 15 & 13 & sim \\
\hline Extensions & 837 & 14 & não \\
\hline and Accidents & 26 & 15 & sim
\end{tabular}

Quadro 4: Resumo dos dados mais relevantes associados ao motor de busca Web of Science

\begin{tabular}{|c|c|c|c|c|c|}
\hline Data & $\begin{array}{l}\text { Password } \\
1\end{array}$ & $\begin{array}{l}\text { Password } 2 \\
\text { e seguintes, } \\
\text { caso } \\
\text { existam }\end{array}$ & $\begin{array}{l}\mathrm{N}^{\circ} \mathrm{de} \\
\text { documentos } \\
\text { obtidos }\end{array}$ & $\begin{array}{l}\mathbf{N}^{\circ} \mathrm{da} \\
\text { pesquisa }\end{array}$ & $\begin{array}{l}\text { Pesquisa } \\
\text { efetuada } \\
\text { ou não }\end{array}$ \\
\hline \multirow[t]{5}{*}{$2019 / 02 / 25$} & Nails & & 14.686 & 16 & não \\
\hline & & $\mathrm{Gel}$ & 162 & 17 & $\begin{array}{l}\text { Inicialmente } \\
\text { não; depois } \\
\text { sim }\end{array}$ \\
\hline & & and Accidents & 0 & 18 & não \\
\hline & & Extensions & 231 & 19 & não \\
\hline & & and Accidents & 3 & 20 & sim \\
\hline
\end{tabular}

Quadro 5: Resumo dos dados mais relevantes associados à pesquisa no motor de busca RCAAP

\begin{tabular}{llllll} 
Data & Password Critérios & $\begin{array}{l}\mathbf{N}^{\circ} \text { de } \\
\text { documentos } \\
\text { obtidos }\end{array}$ & $\begin{array}{l}\mathbf{N}^{\circ} \text { da } \\
\text { pesquisa }\end{array}$ & $\begin{array}{l}\text { Pesquisa } \\
\text { efetuada } \\
\text { ou não }\end{array}$ \\
\hline $2019 / 02 / 24$ & $\begin{array}{l}\text { Unhas de } \\
\text { gel }\end{array}$ & $\begin{array}{l}\text { Pesquisa } \\
\text { avançada; }\end{array}$ & 0 & 21 & não \\
\cline { 2 - 5 } & $\begin{array}{l}\text { Extensões } \\
\text { de unhas }\end{array}$ & 0 & 22 & não \\
\cline { 2 - 5 }
\end{tabular}

Quadro 6: Resumo dos dados mais relevantes associados à pesquisa no motor de busca EBSCO (CINALH, Medline, Database of Abstracts and Reviews, Central Register of Controlled Trials, Cochrane Database of Systematic Reviews, Nursing \& Allied Health Collection $e$ MedicLatina) 


\begin{tabular}{lllll} 
Data & Password & $\begin{array}{l}\mathbf{N}^{\circ} \text { de documentos } \\
\text { obtidos }\end{array}$ & $\begin{array}{l}\mathbf{N}^{\circ} \text { da } \\
\text { pesquisa }\end{array}$ & $\begin{array}{l}\text { Pesquisa efetuada } \\
\text { ou não }\end{array}$ \\
\hline 2019/02/24 & Gel nails & 19 & 23 & $\operatorname{sim}$ \\
\cline { 2 - 5 } & $\begin{array}{l}\text { Nail } \\
\text { extensions }\end{array}$ & 3 & 24 & $\operatorname{sim}$ \\
\end{tabular}

Contudo, nenhum dos artigos selecionados nas pesquisas anteriores foi capaz de responder à questão de investigação; todos os documentos se referiam a assuntos ortopédicos (pelos termos usados em inglês), ao risco existentes nos profissionais que elaboram as unhas de gel (que serão alvo de uma scoping review a realizar a médio prazo) e os eventuais riscos a nível de higiene e desempenho das profissionais de saúde que usem estes adereços (mas sem a dimensão da sinistralidade).

\section{DISCUSSÃO E PERSPETIVAS/ PROJETOS PARA FUTURO}

Uma vez que não se encontraram quaisquer dados sobre este tema, a Direção da Revista Portuguesa de Saúde Ocupacional lança mais um desafio aos seus leitores, no sentido de tentar aliciar que uma Equipa de Saúde Ocupacional (entre Médicos do Trabalho, Enfermeiros do Trabalho, Técnicos de Segurança, Recursos Humanos/ Chefias/ Empregador), que tenham acesso a uma amostra de trabalhadoras significativa, em postos de trabalho adequados a esta investigação. Pretender-se-ia tentar correlacionar o número e gravidade dos Acidentes de Trabalho ao longo dos últimos anos, nas trabalhadoras do sexo feminino, que usem unhas de gel, versus trabalhadoras com tarefas equivalentes, mas sem esses adereços; podendo fazê-lo com total autonomia (desde a recolha das autorizações, elaboração do instrumento para recolha de dados, inserção dos mesmos em SPSS, tratamento estatístico, redação de artigo e submissão para publicação) ou apenas recolhendo as autorizações/ dados e inserindo os mesmos em programa estatístico, assumindo alguns elementos da Direção da Revista as restantes etapas, em parceria.

Existem interessados?!

\section{(1)Mónica Santos}

Licenciada em Medicina; Especialista em Medicina Geral e Familiar; Mestre em Ciências do Desporto; Especialista em Medicina do Trabalho e Doutoranda em Segurança e Saúde Ocupacionais, na Faculdade de Engenharia da Universidade do Porto. Presentemente a exercer nas empresas Medicisforma, Servinecra e Securilabor; Diretora Clínica das empresas Quercia e Gliese; Diretora da Revista Portuguesa de Saúde Ocupacional on line. Endereços para correspondência: Rua Agostinho Fernando Oliveira Guedes, 42, 4420-009 Gondomar. E-mail: s_monica_santos@hotmail.com. 
Doutorado em Enfermagem; Mestre em Enfermagem Avançada; Especialista em Enfermagem Comunitária; Pós-graduado em Supervisão Clínica e em Sistemas de Informação em Enfermagem; Docente na Escola de Enfermagem (Porto), Instituto da Ciências da Saúde da Universidade Católica Portuguesa; Diretor Adjunto da Revista Portuguesa de Saúde Ocupacional on line. 4420-009 Gondomar. E-mail: aalmeida@porto.ucp.pt. Orcid nº 0000-0002-5329-0625.

\section{(3)Catarina Lopes}

Licenciada em Enfermagem, desde 2010, pela Escola Superior de Saúde Vale do Ave. A exercer funções na área da Saúde Ocupacional desde 2011 como Enfermeira do trabalho autorizada pela Direção Geral de Saúde, tendo sido a responsável pela gestão do departamento de Saúde Ocupacional de uma empresa prestadora de serviços externos durante 7 anos. Atualmente acumula funções como Enfermeira de Saúde Ocupacional e exerce como Enfermeira Generalista na SNS24. Encontrase a frequentar o curso Técnico Superior de Segurança do Trabalho. 4715-028. Braga. E-mail: catarinafflopes@gmail.com

\section{(4)Tiago Oliveira}

Licenciado em Enfermagem pela Universidade Católica Portuguesa. Frequenta o curso de Técnico Superior de Segurança no Trabalho. Atualmente exerce a tempo inteiro como Enfermeiro do Trabalho. No âmbito desportivo desenvolveu competências no exercício de funções de Coordenador Comercial na empresa Academia Fitness Center, assim como de Enfermeiro pelo clube de futebol União Desportiva Valonguense. 4435-718 Baguim do Monte. E-mail: tiago_sc16@hotmail.com. 